\title{
Modelling the Formation of 'Type B' Ejecta - The Unique Thermometers of the Fukushima Nuclear Disaster
}

\author{
Lior A.S. Carno \\ University of Bristol \\ Jack J. Turner \\ University of Bristol
}

Peter G. Martin ( $\square$ peter.martin@bristol.ac.uk)

University of Bristol

\section{Research Article}

Keywords:

Posted Date: February 8th, 2022

DOI: https://doi.org/10.21203/rs.3.rs-1257051/v1

License: (c) (i) This work is licensed under a Creative Commons Attribution 4.0 International License.

Read Full License 


\title{
Modelling the Formation of 'Type B' Ejecta - the Unique Thermometers of the Fukushima Nuclear Disaster
}

\author{
Lior A. S. Carno ${ }^{1, *,+}$, Jack J. Turner ${ }^{1, *,+}$, and Peter G. Martin ${ }^{2, *}$ \\ ${ }^{1}$ University of Bristol, School of Physics, HH Wills Physics Laboratory, Tyndall Avenue, Bristol, BS8 1TL, UK \\ ${ }^{2}$ University of Bristol, Interface Analysis Centre, School of Physics, HH Wills Physics Laboratory, Tyndall Avenue, \\ Bristol, BS8 1TL, UK \\ *Ic17812@alumni.bristol.ac.uk, jt17874@alumni.bristol.ac.uk, peter.martin@bristol.ac.uk \\ +these authors contributed equally to this work
}

\begin{abstract}
For the first time, a model was developed to simulate the cooling of the Fukushima Daiichi Nuclear Power Plant reactor Unit 1-derived, 'Type B' radiocaesium bearing microparticles, distributed into the environment during the 2011 nuclear meltdown. By establishing an analogy between 'Type B' CsMP and volcanic pyroclasts, the presented model simulates the rapid cooling of an effervescent silicate melt fragment upon atmospheric release. The model successfully reproduced the bi-modal distribution of internal void diameters observed in 'Type B' CsMP, however, discrepancies in the peak frequencies resulted primarily due to the neglection of internal void coalescence. The model was subsequently utilised to estimate the temperature within reactor Unit 1 in the instant preceding the hydrogen explosion, to be between 1,850 and 1,920 K. Such a model demonstrates the accuracy of the volcanic pyroclast - 'Type B' CsMP analogue, and confirms radial variations in cooling rate as the cause of the vesicular texture of Unit 1 ejecta. The presented findings provide scope to further explore the comparison between volcanic pyroclasts and 'Type B' CsMP via experimentation, which will provide a deeper understanding of the specific conditions within reactor Unit 1 during the catastrophic meltdown at the Japanese coastal plant.
\end{abstract}

\section{Introduction}

On the $11^{\text {th }}$ March 2011, the magnitude 9.0 Great Tōhoku Earthquake occurred off the eastern coast of Japan. The resultant tsunami inundated $560 \mathrm{~km}^{2}$ of land, destroying over a million buildings and killing approximately 19,000 people $\mathrm{e}^{1,2}$. The economic damage was estimated at US\$235 billion, making it the most costly environmental disaster in history ${ }^{3}$. Located 180 $\mathrm{km}$ from the earthquake's epicentre, the Fukushima Daiichi Nuclear Power Plant (FDNPP) comprised six boiling water reactors, shown schematically in Figure 1, operated by Tokyo Electric Power Company (TEPCO). Upon detection of the earthquake at 14:46 Japan Standard Time (JST) ${ }^{4}$, all three operational reactors at FDNPP, Units 1, 2 and 3 (Units 4, 5, 6 were offline at the time), immediately shut down via insertion of fission-inhibiting control rods (also known as safety control rod axe man 'SCRAM'). While the station proved robust seismically, the earthquake damaged off-site power transmission infrastructure, forcing the plant to switch to emergency diesel generators. These failed 40 minutes later, when the entire site was inundated by a $15 \mathrm{~m}$ high tsunami wave, resulting in a station-wide power loss ${ }^{5}$. Additionally, the seawater pumps, residual heat removal systems and electrical switch gear were all destroyed by the tsunami, disabling all of the plants core-cooling capabilities. One hour after 'SCRAM', the three operational reactors were still producing approximately $1.5 \%$ of their nominal thermal output via fission product decay ${ }^{2}$. Isolated from their ultimate heat-sink, the temperature and pressure within the reactor pressure vessels (RPV) rapidly increased, yielding large quantities of steam. In addition, the exothermic interaction of zirconium cladding with this super-heated steam produced an estimated $130 \mathrm{~kg}$ of hydrogen in reactor Unit $1^{6}$. Various attempts to alleviate the growing pressure and cool each of the reactor cores progressively failed, culminating in core meltdowns. On $12^{\text {th }}$ and $14^{\text {th }}$ March, hydrogen explosions occurred in FDNPP Units 1 and 3, respectively, blowing the roofs from both reactor buildings. Reactor Unit 4, despite not being in operation, also exploded due to an influx of the combustible gas being vented from the nearby reactor Unit $3^{7}$. Although the Unit 2 reactor building did not explode, on $15^{\text {th }}$ March its primary containment vessel (PCV) developed a leak ${ }^{2}$, releasing the incident's largest contribution of on-land radioactive contamination ${ }^{8}$.

The FDNPP accident, rated 7, the highest level, on the International Nuclear and Radiological Event Scale (INES), released an estimated total of 340-800 PBq of radioactivity to the surrounding environment. This was approximately one tenth of the radiation released during the 1986 Chernobyl Nuclear Power Plant disaster ${ }^{9}$. Cumulative releases from the three FDNPP reactor units, combined with their intermittent cooling histories, resulted in a diverse suite of emissions of far greater complexity 


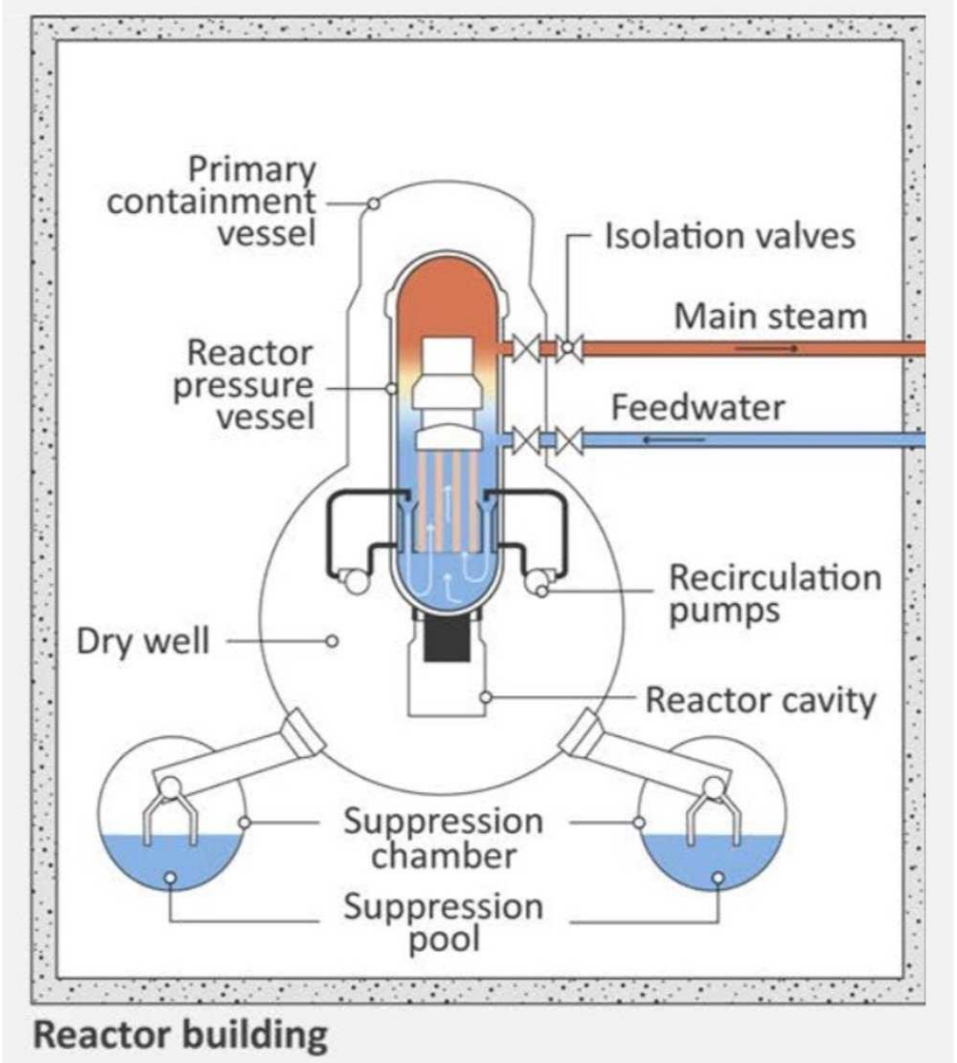

Figure 1. Schematic of the Mark-I containment associated with the boiling water reactors (BWR) used in FDNPP Units 1-5. Water is used both as coolant and as a neutron moderator (via its flow rate) alongside neutron absorbing control rods to control reactivity. Pure uranium oxide $\left(\mathrm{UO}_{2}\right)$ was used as the nuclear fuel in reactor Units 1 and 2, while a component of mixed oxide $(\mathrm{MOX})$ was used in reactor Unit $3^{2}$. These fuel elements are encased within zirconium cladding (Zircaloy-4 ${ }^{\mathrm{TM}}$ ), and the primary containment assembly and pipes for the heat exchanger network are clad with a Rockwool ${ }^{\mathrm{TM}}$ type insulation. Adapted from ${ }^{4}$.

than the single release episode during the Chernobyl disaster ${ }^{8}$. The radionuclides released, which spread throughout Japan, as seen in Figure 2, consisted mainly of the radiocaesium isotopes, ${ }^{134} \mathrm{Cs}$ and ${ }^{137} \mathrm{Cs}$, with half-lives of 2.06 and 30.07 years, respectively, and radioiodine, ${ }^{131} \mathrm{I}$, with a considerably shorter half-life of 8.02 days ${ }^{10}$. Due to its 8 day half-life, the latter quickly decayed out of the environment, leaving radiocaesium as the primary gamma-emitting pollutant.

Following the accident, a soluble form of radiocaesium was widely detected in the surrounding soils, rivers and plants ${ }^{11}$. The insoluble form of Fukushima-derived radiocaesium was first identified in the environment two years after the accident by Adachi et al. ${ }^{12}$. Termed 'caesium-bearing microparticles' (CsMP), these micron-scale particles are $\mathrm{SiO}_{2}$-based with a high specific radioactivity. Their glassy-state make them resistant to erosion processes, meaning they present a far greater sustained radiation hazard than the soluble form of radiocaesium ${ }^{13,14}$. Because CsMP were formed inside the reactors during the FDNPP accident, their properties provide critical insight into the meltdown chronology and conditions ${ }^{15}$. The study of CsMP is therefore vital for assessing the extent of reactor damage and planning for their decommissioning, as well as to the cleanup of the contaminated areas surrounding FDNPP.

CsMP have been broadly classified into two groups: 'Type A' and 'Type B', originating from reactor Unit 2 and Unit 1, respectively ${ }^{16,17}$. These groups are characterised primarily by their varying ${ }^{134} \mathrm{Cs} /{ }^{137} \mathrm{Cs}$ activity ratio, which arises due to the differing fuel burn-up from each reactor. Via comparison of these measured ${ }^{134} \mathrm{Cs} /{ }^{137} \mathrm{Cs}$ ratios with those calculated analytically using reactor-core inventory modelling ${ }^{18}$, the source reactors of 'Type A' and 'Type B' CsMP have been successfully identified. In addition to their $\mathrm{Cs}$ activity ratio, the two CsMP types are distinguishable by their morphology. 'Type A' particles are smaller, ranging in size from 2-10 $\mu \mathrm{m}$ and highly spherical, thus are commonly termed 'Cs-balls' ${ }^{2}$. In contrast, 'Type B' material is larger, with particle diameters ranging from $50-400 \mu \mathrm{m}^{16}$, and generally more angular, although spherical suites of 'Type B' particles have been identified ${ }^{19}$. Moreover, different CsMP are prevalent in certain locations; 'Type A' particles in the western regions and 'Type B' particles in the northern areas close to the FDNPP site ${ }^{16}$. A summary of the differences between 'Type A' and 'Type B' particles is given in Table 1. Due to their narrow spatial distribution and proximity to the FDNPP site $(\leq 8 \mathrm{~km})$, 


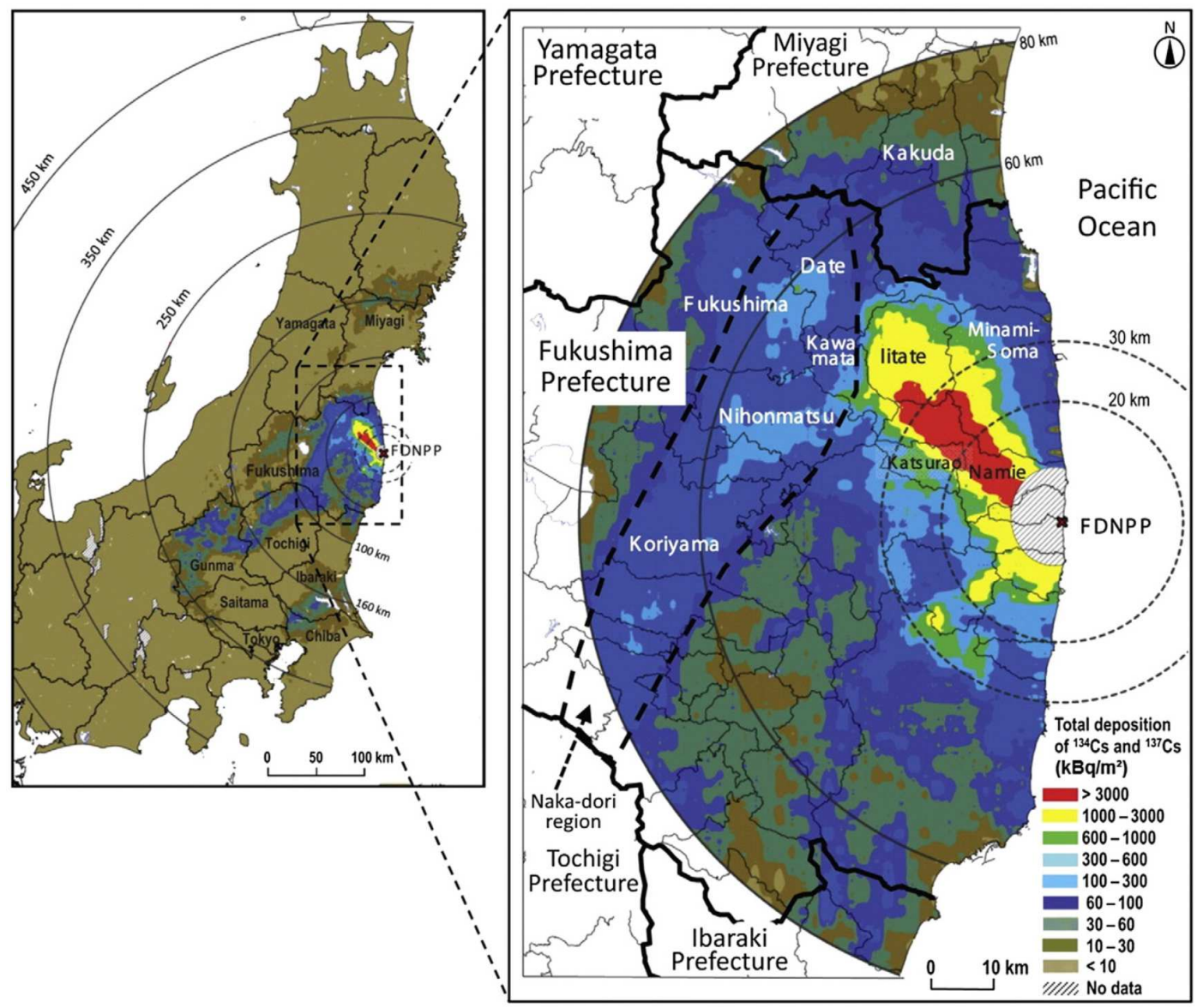

Figure 2. Estimated total distribution of radiocaesium after the FDNPP accident. The narrow red band corresponds to the highest activity region of the primary containment plume that was released north west from Unit 2 of the FDNPP ${ }^{9}$. Adapted from $^{20}$.

\begin{tabular}{|l|l|l|}
\hline Characteristic & 'Type A' & 'Type B' \\
\hline Size Distribution (observed) & $1-10 \mu \mathrm{m}$ & $70-400 \mu \mathrm{m}$ \\
\hline Cs Activity Ratio (mean) & 1.04 & 0.93 \\
\hline Source Reactor(s) & Unit $2 / 3$ & Unit 1 \\
\hline Emission Date (estimate) & March $15^{\text {th }}, 2011$ & March $12^{\text {th }}, 2011$ \\
\hline${ }^{137}$ Cs Activity (Bq/particle) & $\sim 10^{-2}-10^{2}$ & $\sim 10^{1}-10^{4}$ \\
\hline Geographical Distribution & Wide & Limited \\
\hline
\end{tabular}

Table 1. Comparison of 'Type A' and 'Type B' particle properties. Adapted from ${ }^{16,19}$.

obtaining large numbers of such 'Type B' particles has proved challenging. As a result, there exists limited research regarding 'Type B' material in contrast with the comprehensively studied 'Type A' particles (see e.g. ${ }^{11}$ ). For this reason, the chosen focus of this research is the formation of 'Type B' CsMP, rather than the more abundant 'Type A' material.

Energy Dispersive Spectroscopy (EDS) analysis has identified Si as the primary constituent (24.9-37.1 wt.\%) of 'Type B' CsMP, whilst synchrotron characterization has revealed a highly heterogeneous distribution of other elemental constituents (including $\mathrm{Mo}, \mathrm{Fe}, \mathrm{Ni}, \mathrm{Cd}, \mathrm{Sn}$, and $\mathrm{Cr})^{16,21,22}$. Owing to this primarily $\mathrm{Si}$ based composition, 'Type B' CsMP have been invoked to originate from the fibrous (Si-based) Rockwool ${ }^{\mathrm{TM}}$ thermal insulation found in abundance throughout FDNPP, namely surrounding the RPV ${ }^{16}$. Artefacts of this fibrous material are observed as inclusions on the surface of 'Type B' CsMP, as highlighted in Figure 3D. These exhibit a uniform orientation across the particle surface, suggesting the CsMP are the result of a violent emission event such as the hydrogen explosion that occurred on $12^{\text {th }}$ March $2011^{22}$.

Initial examination using scanning electron microscopy (SEM) showed the surface of 'Type B' CsMP consisted of smooth 


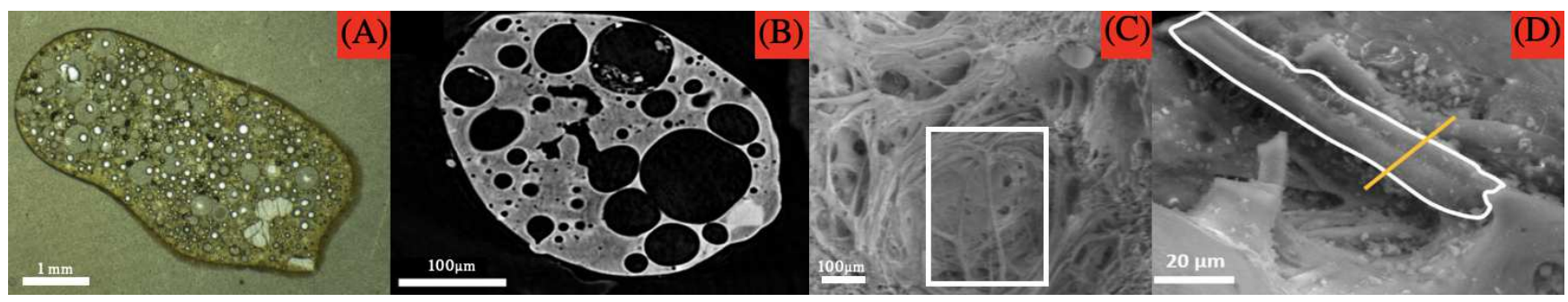

Figure 3. (A) Photomicrograph of a 'Pele's tear,' cut along the direction of elongation, showing highly spherical, internal vesicles which decrease in size towards the particle's edge, from ${ }^{23}$. (B) Orthogonal X-ray tomographic (absorption contrast) section of a 'Type B' CsMP displaying the presence of many, different sized voids within the particle, comparable in its internal structure to (A), from ${ }^{21}$. (C) Scanning electron microscope (SEM) image of the internal structure of volcanic pumice, revealing fibrous features (marked by the white square), from ${ }^{24}$. (D) SEM image of the surface of 'Type B' CsMP, marked are the fibrous features, akin to the fibrous features observed in $(\mathrm{C})$, from $^{22}$

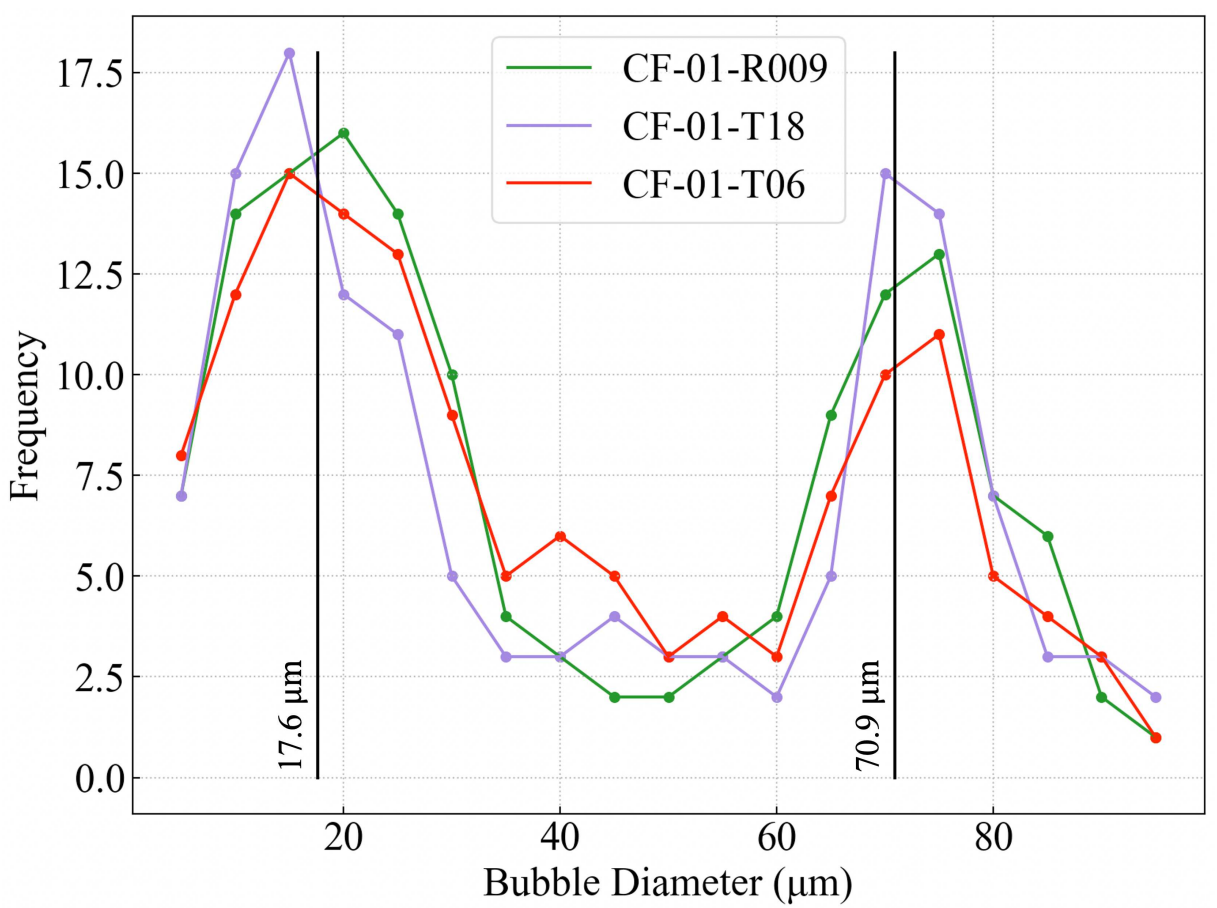

Figure 4. Plot of void diameter against frequency demonstrating the bi-modal void size distribution within 'Type B' CsMP. The first peak, located at $17.6 \mu \mathrm{m}$, represents the incorporated fission product bubbles, 'frozen' around the particle circumference due to quench-like cooling. The second peak, centred at $70.9 \mu \mathrm{m}$, shows the increased diameter of the gaseous voids as a result of depressurisation and coalescence. Adapted from ${ }^{21}$.

sections interrupted by numerous micron-scale spherical voids ${ }^{16,22}$. More detailed investigations into the internal 3D structure via SR- $\mu$-XRF and X-ray tomography (XRT) identified a significant internal volume (24 - 31\%) of spherical voids which display a bi-modal diameter distribution, as shown in Figure 4. ${ }^{19,21,25}$. The smaller voids, with a mean diameter $=17.6 \mu \mathrm{m}$, are concentrated around the circumference of CsMP and are believed to be caused by the incorporation of the prevalent gas, within the reactor, composed of fission products, noble gases and hydrogen, into the molten silicate due to the considerable overpressure of this gas. In contrast, the larger, more centrally located voids, with a mean diameter $=70.9 \mu \mathrm{m}$, likely originate from the release of trapped volatile species from silicate fibres into the bulk particle ${ }^{21}$. Such a bi-modal void diameter distribution was presumably caused by the radially varying cooling rate within the CsMP. Following the hydrogen explosion of reactor Unit 1 , molten CsMP were ejected from the PCV, where they formed, into the environment. Upon exposure to the atmosphere, the CsMP surfaces were cooled almost instantly. This caused the exterior silicate melt to solidify, creating a porous outer rind with voids 'frozen' in place. The internal particle bulk cooled at a somewhat slower rate, allowing for void coalescence as well as 
significant expansion of the more centrally located voids owing to depressurisation ${ }^{21}$.

To date, no studies have been conducted which investigate the formation mechanisms of 'Type B' CsMP. However, the formation of volcanic ejecta material, specifically pyroclastic bombs, is well documented and multiple studies have successfully used the thermal history of these ejecta to examine eruption dynamics ${ }^{26-28}$. Such volcanic bombs are found as ballistically emplaced clasts in the crater or on the flank of volcanoes or in pyrcoclastic flow deposits kilometers away from the vents (Guagua Pichincha ${ }^{27}$; Montserrat ${ }^{28}$; Tungurahua ${ }^{29,30}$ ). Despite being several orders of magnitude larger than 'Type B' CsMP, volcanic bombs share many key properties with the Unit 1 particulate released in the Fukushima disaster. Like 'Type B' CsMP, this form of pyroclast starts as part of an effervescent mass of silicate melt within the magmatic shaft of a volcano. During explosive eruptions, this bulk is ejected into the atmosphere as small fragments of melt that experience rapid cooling and depressurisation $^{31}$. During this phase, volatile exsolution and bubble growth commence, but are quickly slowed by the increase in magmatic viscosity of the melt. The glass transition temperature, $T_{g}$, is the kinetic limit at which a material transitions from a viscous liquid to a glass ${ }^{32}$. Different depths within the clast cross $T_{g}$ at different times, resulting in a radial bubble size distribution that provides a textural record of the clast's thermal history. The final morphology of solidified ejecta from both FDNPP Unit 1 and volcanic eruptions are closely related. Both are composed primarily of silicate; $64-69 \mathrm{wt} . \%$ in 'Type B' CsMP and $\sim 56-65 \mathrm{wt} . \%$ in volcanic bombs ${ }^{30,33}$. Moreover, the vesicular interior surrounded by a dense rind of small bubbles observed in the pyroclasts is also very similar to the morphology of 'Type B' CsMP, as demonstrated in Figure 3.

We therefore hypothesise that 'Type B' CsMP experience identical formation mechanisms to volcanic bombs ${ }^{26,34,35}$. The extensive implementation of this volcanic bomb analogy enables the utilisation of volcanology research, as well as the adaptation of volcanic bomb cooling models, to construct a simulation of 'Type B' CsMP cooling. This is subsequently used to further investigate CsMP material properties and hence aid their removal from the environment, as well as estimate the conditions within reactor Unit 1 during the FDNPP accident.

\section{Results}

\section{Parameter Selection}

A complete list of the parameters used in our model is provided in Table 2. The selected parameters were chosen to mimic the properties of CsMP and their surrounding environment: the thermal conductivity, heat capacity and density of 'Type B' particulate were taken to be the same as those of volcanic bombs. It was assumed the particles were carried by the explosive shockwave, thus had the same velocity, which was determined from CCTV footage of the blast ${ }^{6}$. The average elemental composition of the CsMP was obtained from ${ }^{22,33,36}$. These weight percentages, combined with the optimisation parameters $\mathrm{in}^{37}$, were then used to calculate the parameters $B$ and $C$ and calibrate the viscosity model. Since the particles were released into the surrounding environment after the hydrogen explosion, the external pressure experienced by the particle was taken to be atmospheric pressure. Considering the particles were in the reactor for approximately one day before the explosion, it was assumed they had reached thermal equilibrium with their surroundings. Therefore, the initial pressure of the bubbles within the CsMP was taken to be the estimated pressure of the PCV (the vessel where the CsMP formed) before the hydrogen explosion occurred. As bubbles close to the melt exterior experienced insignificant levels of growth in real 'Type B' CsMP, the initial radii of the modelled voids were randomly sampled from the observed distribution of circumferential void diameters. Finally, the external temperature and air velocity were obtained from weather reports of Fukushima on $12^{\text {th }}$ March 2011, whilst the air viscosity and conductivity were assumed to be that of ambient air.

\section{Void Diameter Distribution}

The simulated void diameter distribution for three simulated CsMP of different sizes is shown in Figure 5. Comparable to the observed void diameter distribution, shown in Figure 4, a bi-modal distribution is also evident, indicating the model correctly reproduced the cooling profile which occurred in actual 'Type B' CsMP. The first peak is centred, on average, at 17.6 $\mu \mathrm{m}$, identical to the position of the first peak in Figure 4. However, the second peak appears at a higher frequency and lower void diameter than the observed data. The likely reasons for this discrepancy are subsequently discussed.

\section{Temperature Estimate}

Via investigation of void growth at various initial temperatures, the temperature of reactor Unit 1 was estimated to be between $1,850-1,920 \mathrm{~K}$, marked by region B in Figure 6. This estimate was established by varying the initial temperature of the modelled CsMP and determining the effects on void growth. With reference to Figure 6, at temperatures above 1,920K (region $\mathrm{C}$ ), the growth of surface level bubbles was significant. In reality, the surface level bubbles experienced negligible growth due to quench-like cooling. Hence, any temperature corresponding to a modelled situation where surface level voids grew was unrealistic, so was deemed an invalid estimate. The lower temperature bound was established to be the temperature at which negligible central bubble growth occurred, and the error in final bubble diameter was taken to be the range of bubble diameters obtained when using the maximum and minimum values of the calibration parameters. 


\begin{tabular}{|l|l|l|l|l|}
\hline Description & Symbol & Value & Unit & Reference \\
\hline & & Particle Parameters & & \\
Thermal conductivity & $k_{b}$ & 1.5 & $\mathrm{Wm}^{-1} \mathrm{~K}^{-1}$ & 26 \\
Heat capacity & $c_{b}$ & 1096 & $\mathrm{Jkg}^{-1} \mathrm{~K}^{-1}$ & 26 \\
Density & $\rho_{b}$ & 2400 & $\mathrm{kgm}^{-3}$ & 26 \\
Particle velocity & $v_{b}$ & 360 & $\mathrm{~ms}^{-1}$ & 6 \\
\hline & & Viscosity Parameters & & \\
Fit parameter & $A$ & -4.55 & - & 37 \\
Fit parameter & $B$ & 4583.1 & $\mathrm{~J}$ & - \\
Fit parameter & $\mathrm{C}$ & 674.5 & $\mathrm{~K}$ & - \\
Glass transition temperature & $T_{g}$ & 951.4 & $K$ & - \\
\hline & & Bubble Parameters & & \\
External Pressure & $P_{e}$ & $0.1(1$ atm) & $\mathrm{MPa}$ & 38 \\
Internal Pressure & $P_{0}$ & 0.55 & $\mathrm{MPa}$ & 5 \\
Initial void radius & $R_{0}$ & random (<10) & $\mu \mathrm{m}$ & 21 \\
\hline & & Air Parameters & & \\
Prandtl number & $\mathrm{Pr}$ & 0.71 & - & 26 \\
External (air) temperature & $T_{\infty}$ & 280 & $K$ & 39 \\
Air viscosity & $\eta_{g}$ & $1.71 \times 10^{-5}$ & $\mathrm{~Pa} \mathrm{~s}$ & 27 \\
Air velocity & $v_{g}$ & 1.6 & $\mathrm{~ms}$ & 39 \\
Air conductivity & $k_{g}$ & 0.024 & $\mathrm{Wm} \mathrm{K}^{-1} \mathrm{~K}^{-1}$ & 34 \\
\hline
\end{tabular}

Table 2. Parameter values utilised in the presented model.

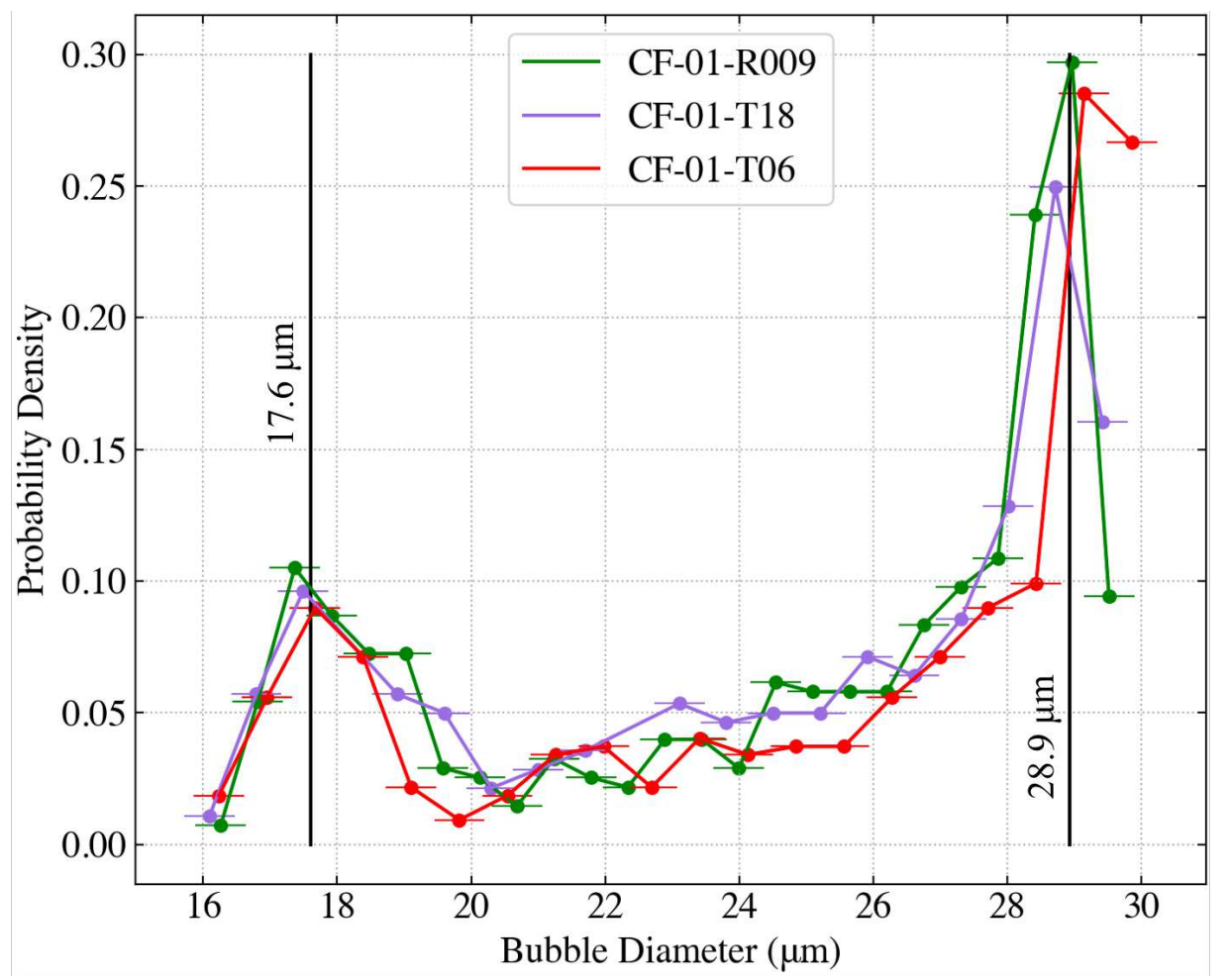

Figure 5. Apparent bi-modal void diameter distribution within the simulated particles comparable to CF-01-R009, CF-01-T18 and CF-01-T06 with respective diameters of $406.5 \mu \mathrm{m}, 336.5 \mu \mathrm{m}$ and $384.5 \mu \mathrm{m}$. The observed distribution was obtained using an initial pressure of $0.55 \mathrm{MPa}$ and an initial temperature of $1,900 \mathrm{~K}$ and shows peaks centred around $17.6 \mu \mathrm{m}$ and $28.9 \mu \mathrm{m}$, respectively. The errors were caused by the range of bubble diameters produced across the range of calibration parameters. 


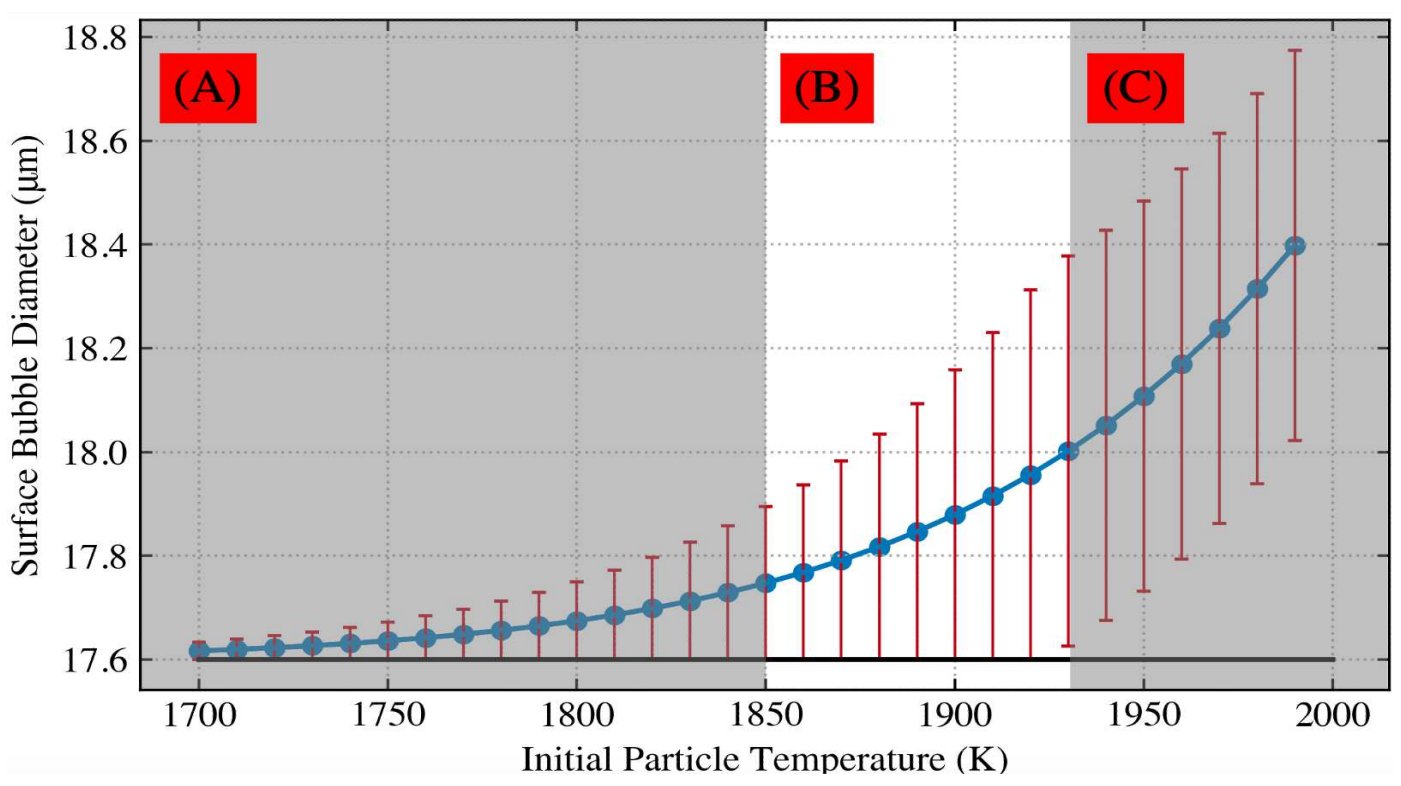

Figure 6. Plot of of surface bubble diameter against initial particle temperature used to estimate the temperature within reactor Unit 1. Region A marks the temperatures which were deemed too low, due to negligible central void growth, whereas region $\mathrm{C}$ marks those deemed too high, due to excess growth of surface level voids. Region B, however, marks the obtained estimated temperature range. The resulting errors are from the range of bubble diameters produced across the range of calibration parameters.

\section{Discussion}

In order to minimise the complexity of the presented model, several assumptions were made. First, the assumption that CsMP were spherical and isotropic allowed for the reduction of a 3-dimensional problem into that of only 1 dimension. Many 'Type B' CsMP were well rounded by surface tension forces during transport and have aspect ratios close to $1^{33}$, thereby validating this approximation. However, irregularly-shaped 'Type B' particles have also been identified in the environment ${ }^{8}$, whose cooling was less accurately captured by our model. Due to their higher surface area, cooling of these more angular particles would have been faster ${ }^{40}$. As a result, they would be expected to have a higher number of surface level voids with smaller diameters due to the increased surface area and faster cooling.

Secondly, the modelled CsMP were assumed to cool via forced convection only. The py-pde python package ${ }^{41}$, which implicitly evaluates partial differential equations, was utilised to verify this assumption via calculation of surface heat flux due to both convective and radiative cooling. These calculations confirmed radiative CsMP cooling was negligible, and therefore that the initial assumption was valid. In fact, the radiative cooling contribution was even smaller than that calculated, since the calculation assumed CsMP were emitting as black bodies and consequently outputting the maximum radiative heat flux. This second assumption greatly reduced the computational complexity of the model, as it enabled the use of the faster analytical solution as opposed to the inefficient implicit solver.

In addition, it was assumed that the melt viscosity was uniform across the bubble-melt interface. In reality, the radial temperature gradient resulted in a higher melt viscosity at the outermost edge of a bubble than at the innermost edge. This variation was found to be negligible on the scale of even the largest voids, and therefore allowed the viscosity value of the melt shell to be taken as the viscosity corresponding to the co-ordinate of the bubble centre.

Furthermore, the model did not also account for the effects of crystal structure formation which, if present, acts to increase melt viscosity and restrict bubble growth. In volcanology, these effects are accounted for using the Einstein-Roscoe correlation ${ }^{26}$. However, for 'Type B' CsMP, the cooling timescale is much shorter than the crystallisation timescale ${ }^{42}$. Hence, while there may be a high level of nucleation within the 'Type B' CsMP, there is little-to-no crystal growth so the omission of such effects was accurate.

Finally, there was assumed to be a constant molar mass of gas inside growing bubbles. In fact, Martin et al. ${ }^{21}$, detected bright 'halos' of increased X-ray attenuation in EDS measurements caused by local differences in volatile (fission product) elements. These indicate that as bubbles grew during decompression, volatiles diffused in from the surrounding melt and were subsequently resorbed as the temperature dropped and the melt solubility increased causing Cs and Sr enrichment at the bubble margin. As a result, the number of moles of internal gas changed marginally during bubble growth. This was not accounted for 
in this work, but future iterations of the model should take these processes into account in order to elucidate refined temperature and pressure regimes for 'Type B' CsMP formation. For example, the equations developed by Prousevitch et al. ${ }^{48}$, account for bubble growth due to a changing volatile concentration profile, which itself might be inferred from the concentrations of different volatile species in and around voids at different particle depths.

As demonstrated in Figure 5, the presented model successfully reproduced a bi-modal void diameter distribution, comparable to that observed in real 'Type B' CsMP (Figure 4). However, the simulation did not account for internal void coalescence, and as a result it was not possible to match the relative frequency of the two peaks, nor their positions, to the real data. During the rapid depressurisation of a CsMP, internal bubbles grew until eventually adjacent bubbles coalesced and merged, resulting in central voids much larger than the maximum size calculated by our model. This process is visible in the interior of certain 'Type B' CsMP (Figure 7) if the CsMP melt solidified before void coalescence was complete. These interactions between bubbles can be comfortably neglected when examining the formation of porous pyroclasts as the scale of maximally-grown bubbles is several orders of magnitude smaller than the clast size. This is not the case for the micron-scale 'Type B' CsMP. Thus, a complete analysis including coalescence is needed to accurately predict the final radii of the largest bubbles in 'Type B' CsMP. It is expected that this will increase the final radii of large voids whilst reducing their frequency, thereby recovering the void diameter distribution observed in real CsMP.

Regardless, the model presented in this study clearly demonstrates the accuracy of the CsMP-volcanic bomb analogy, and confirms the hypothesis of Martin et al. ${ }^{33}$, who invoked the radial variation in cooling rate as the cause of a bi-modal void diameter distribution. Since the CsMP were molten in the instant preceding their environmental release, the temperature of reactor Unit 1 was expected to exceed the $1,491 \mathrm{~K}$ melting point of the CsMP precursor material ${ }^{43}$ (Rockwool $^{\mathrm{TM}}$ ), as our estimate does. Our temperature estimate is also concordant with the lower bound imposed by the presence of Sb in 'Type B' CsMP, which implies the particles formed at temperatures greater than $1,860 \mathrm{~K}^{16}$, lending further credence to our results. Although temperatures in the reactor were known to be in excess of 3,073 K during the core meltdown, the hydrogen explosion occurred $\sim 8$ hours after this melting reportedly ended. In this time, venting of the suppression pool took place, which would have resulted in the inflow of air to the damaged RPV and PCV, causing the reactor temperature to fall to between those temperatures inferred by our model.

The analogy between 'Type B' CsMP and volcanic glassy airfall implies these reactor Unit 1 derived particulates may be brittle and easily friable, akin to pumice. However, the rapid quenching $(\sim 0.2 \mathrm{~s})$ predicted by our model yields silicates with innate mechanical strength due to high internal residual stresses, very similar to Pele's tears or Prince Rupert's drops ${ }^{23,44}$. In addition, the tendency to minimize the total surface area of the gas-melt interface in the absence of shear (deformational) stresses results in the rounding of melt fragments, which further contributes to CsMP mechanical strength ${ }^{33}$. The characteristic timescale for this rounding process is given by;

$$
\tau_{\text {round }}=\frac{\eta r}{\Gamma}
$$

where $\eta$ is melt viscosity, $r$ melt radius, and $\Gamma$ surface tension (which is of order $10^{-1} \mathrm{~N} \mathrm{~m}^{-1}$ ). For a $200 \mu \mathrm{m}$ particle cooling from $1,900 \mathrm{~K}, \tau_{\text {round }} \sim 0.5 \mathrm{~ms}$. The time for an equally sized particle to cool to below the glass transition is $\sim 10 \mathrm{~ms}$, which proves 'Type B' CsMP experienced significant rounding in the atmosphere before solidification. Their resistance to mechanical attrition invokes that they will be stable in the environment for a sustained period of time. However, the vitrified state of highly active $\mathrm{Cs}$ and $\mathrm{UO}_{2}$ entrapped in the glassy matrix implies that the breakdown of 'Type B' CsMP represents a significant radiation hazard by exposing these elements and compounds ${ }^{25}$. Fortunately, the significant size and strength of the particulates mean they are unlikely to fragment further under surface environmental conditions; contrasted with the much smaller $(1-10 \mu \mathrm{m})$ suite of radioactive 'Type A' material which is easily re-suspended and able to penetrate deep inside the lungs and possibly enter the bloodstream ${ }^{45}$.

TEPCO plans to commence decommissioning and dismantling operations at FDNPP Unit 1 in December 2021, a large part of which includes the mechanical milling and removal (via a robotic arm) of the fuel debris ${ }^{33}$. The Corium 'slump' of fuel and reactor core components melted through both the RPV and the PCV by the time the hydrogen explosion on $12^{\text {th }}$ March, resulting in violent molten core-concrete interactions (MCCI). Comparison of the aforementioned magmatic systems associated with the nuclear meltdown implies that this debris is also likely to be extremely resistant to mechanical breakdown. The proposed removal methods are therefore expected to be significantly more challenging than in other reactor buildings, however, we foresee the risk of radioactive dust generation to be minimal due to mechanical properties of 'Type B' material outlined above.

Following the work of Benage et al. ${ }^{26}$, an improvement of our model upon earlier pyroclast cooling models is the inclusion of the relative velocity between the ejecta material and ambient air in the calculation of the Reynolds number. While out of the scope of this study, an investigation into the effect of the ambient air temperature and velocity on the final CsMP texture would provide useful information about the buoyant air entertainment of particles after the hydrogen explosion. This data could then 


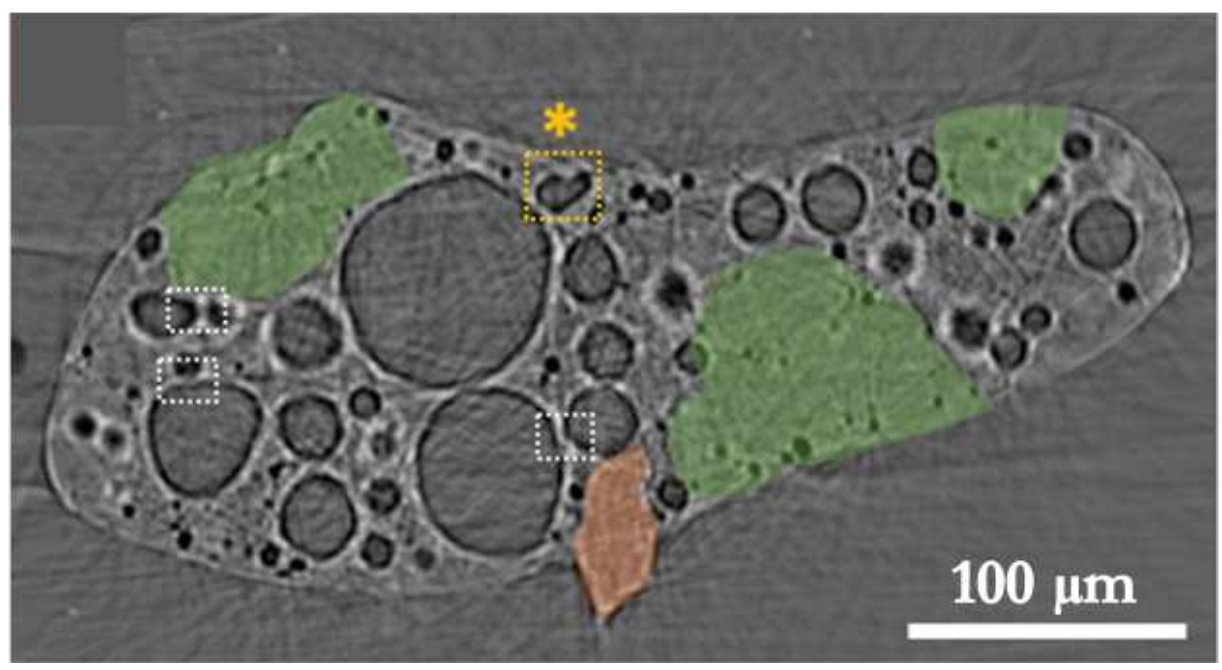

Figure 7. Synchrotron radiation XRT image of the inner structure of a 'Type B' CsMP. Marked by the white and yellow boxes are the locations where voids connect or coalesce, respectively. The Fe-rich region of the particle is highlighted in orange, while the green region marks Ca-rich, low porosity areas. From ${ }^{21}$.

be used to refine existing fallout dispersion models of the accident, such as the work by Yoshida et al. ${ }^{20}$, and identify possible areas of contamination.

The rounding of large 'Type B' CsMP after ejection lends credence to the spherical approximation used in this work. However, a significant number of Unit 1 particulates with irregular shapes have also been found in the environment ${ }^{8}$. In order to more accurately study the formation of this material, it is recommended to couple a commercial finite element heat transfer package (e.g. Dassault Solidworks ${ }^{46}$ ) with the bubble growth model presented herein ${ }^{47,48}$. In addition, 3D XRT data of 'Type B' CsMP could be used to generate the mesh of the proposed simulation, with the actual coordinates of void nuclei being used for the lower-scale bubble growth model rather than the random sampling method utilised in this work.

As is the case with all mathematical modelling of physical systems, experimental validation is still required to confirm the findings of this study. We propose an experiment similar to those conducted by Okumura et al. and Kogure et al. ${ }^{49,50}$, where synthesis of smaller 'Type A' material was attempted in order to elucidate formation mechanisms for the Unit 2 derived particulates:

1. Suspend samples of precursor Rockwool ${ }^{\mathrm{TM}}$ insulation upon a heat resistant pedestal (e.g. ceramic) inside a pressurized, non-reactive cell (stainless steel or zirconium).

2. Connect the cell to a gas rig to control the pressure and the inflow of gases, including tracer gases such as hydrogen or deuterium.

3. Using an internal filament, melt the sample at the temperatures identified in this study.

4. Rapidly cool and depressurise the system using a turbo-pump, thereby quenching the melt.

5. Analyse the resulting material using focused ion beam (FIB), SEM, XRT, and X-ray fluorescence (XRF) techniques and compare the results to real 'Type B' CsMP samples.

\section{Methods}

\section{Model Compilation}

The constructed model simulated a CsMP cooling upon atmospheric release due to the Unit 1 hydrogen explosion. The model was built on two scales: the first being the scale of the particle, where temperature and viscosity changes were modelled, the second being the scale of pore growth. These two models were then coupled to obtain a complete cooling profile of 'Type B' CsMP. A model schematic is shown in Figure 8. 


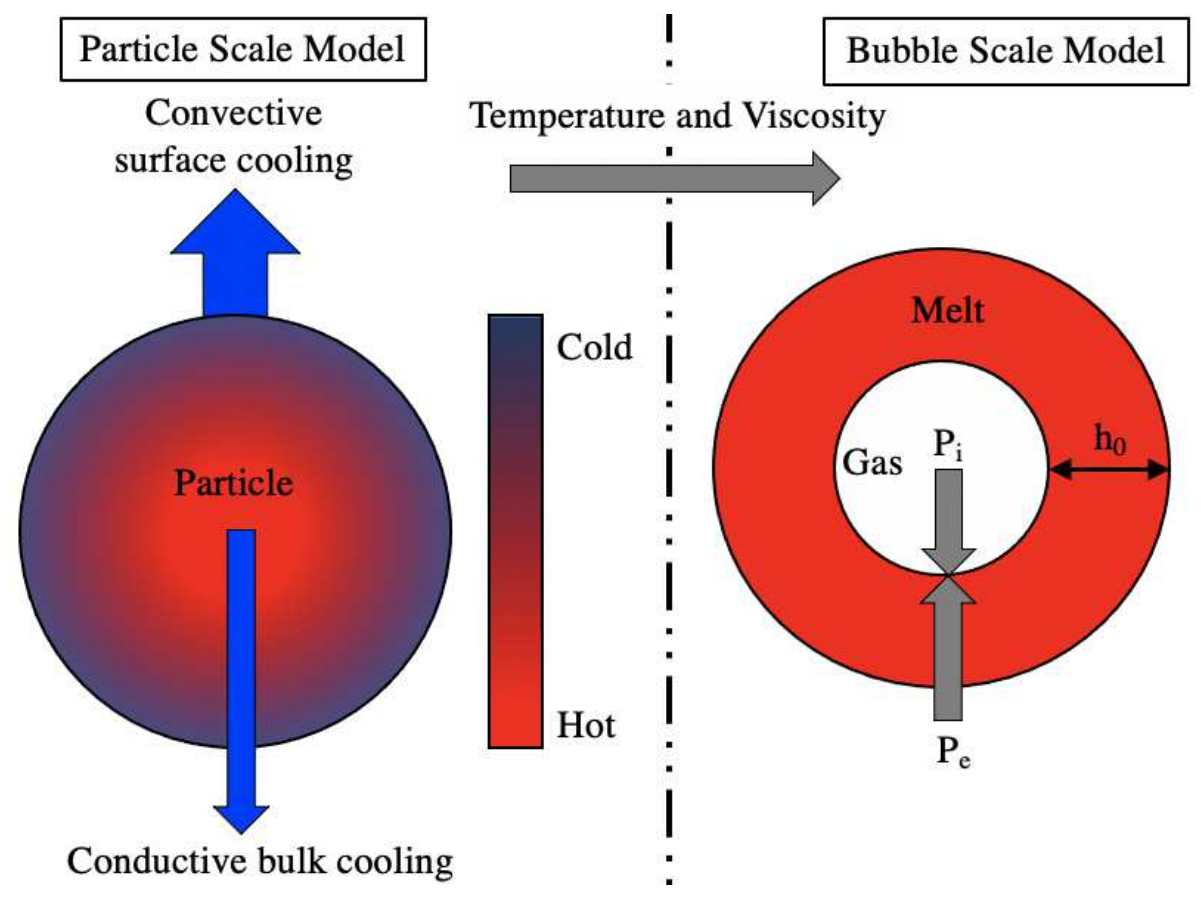

Figure 8. Model schematic outlining the simulated physical processes at each scale of the model.

\section{Particle Cooling}

Upon exposure to the atmosphere, the released particles cooled adiabatically. The cooling of each particle was modelled by assuming convective heat loss occurred only at the particle surface, since the bulk of the particle cooled via conduction due to the heat gradient formed between the centre and the surface of the particle, as opposed to convection ${ }^{51}$. The radial and temporal heat profile of the particle due to this conductive heat loss was modelled by solving the one-dimensional, spherically symmetric heat equation;

$$
\rho_{b} c_{b} \frac{\partial T}{\partial t}=\frac{k_{b}}{r^{2}} \frac{\partial}{\partial r}\left(r^{2} \frac{\partial T}{\partial r}\right),
$$

where $\rho_{b}$ is density of the particle, $c_{b}$ the heat capacity of the particle, $k_{b}$ the thermal conductivity and $r$ the radial coordinate. The physical particle properties (e.g. density, heat capacity, thermal conductivity) were assumed to be constant throughout the model. The convective heat loss at the particle surface was accounted for by imposing the boundary condition;

$$
k_{b}\left(\frac{\partial T}{\partial r}\right)_{r=R}=-q_{c},
$$

where $q_{c}$ is the convective heat flux, calculated by;

$$
q_{c}=h\left(T_{\infty}-T_{s}\right),
$$

where $h$ is the heat transfer coefficient, $T_{s}$ is the surface temperature of the particle and $T_{\infty}$ the temperature of the surroundings ${ }^{23}$. The heat coefficient was calculated using the equation;

$$
h=\frac{N k_{g}}{2 r_{p}},
$$

where $N$ is the Nusselt number, $k_{g}$ the thermal conductivity of the air surrounding the particle and $r_{p}$ the particle radius. Calculation of the Nusselt number required the particle Reynolds number, $R e$, determined by;

$$
R e=\frac{2\left|\left(v_{g}-v_{p}\right)\right| r_{p} \rho_{g}}{\eta_{g}},
$$


where $v_{g}$ is the velocity of the surrounding air, $v_{p}$ the particle velocity, $\rho_{g}$ the air density and $\eta_{g}$ the air viscosity. The Nusselt number was subsequently calculated using;

$$
N=2+x \operatorname{Re}^{\frac{1}{2}} \operatorname{Pr}^{\frac{1}{3}},
$$

where $x=\left(2.2 \times 10^{-4}\right) \rho_{p}+0.31$ and $\operatorname{Pr}$ is the Prandtl number, taken to be 0.71 for ambient air $^{26}$.

\section{Particle Viscosity}

The viscosity of the molten silicate comprising 'Type B' CsMP fluctuated over 15 orders of magnitude whilst the particle cooled. This change was modelled using the empirical Vogel-Fulcher-Tamman (VFT) model;

$$
\log (\eta)=A+\frac{B}{T-C}
$$

where $\eta$ is viscosity, $T$ is temperature and $A, B$ and $C$ are empirically determined constants. The parameter $A$ represents the melt viscosity at an infinite temperature and can, to high degrees of accuracy, be considered independent of melt composition ${ }^{37}$. However, $B$ and $C$ can not and they can be calculated as follows:

$$
\begin{aligned}
& B=\sum_{n=1}^{7}\left[b_{i} M_{i}\right]+\sum_{j=1}^{3}\left[b_{1 j}\left(M 1_{1 j} \cdot M 2_{1 j}\right)\right], \\
& C=\sum_{n=1}^{6}\left[c_{i} N_{i}\right]+\left[c_{11}\left(N 1_{11} \cdot N 2_{11}\right)\right] .
\end{aligned}
$$

$M$ and $N$ represent the weight percentage of a given chemical component and $b$ and $c$ are empirically determined optimization parameters $^{37}$.

\section{Void Growth}

The voids within 'Type B' material grew as the particulate cooled. Therefore, bubble size and position provide valuable insight into the cooling history of the CsMP. Since the bubbles were assumed to be perfectly spherical, radial bubble growth was modelled in one-dimension. By assuming the bubble growth was limited by the viscosity of a thin shell of surrounding melt only, radial growth was described using the equation;

$$
\frac{d R}{d t}=\frac{R^{2}}{12 \eta h}\left(P_{i}-P_{e}\right)
$$

where $R$ is bubble radius, $t$ time, $\eta$ the viscosity of the surrounding melt, $h$ the shell thickness, $P_{i}$ the outwardly directed internal bubble pressure and $P_{e}$ the external pressure experienced by the bubble. The full analysis of the expansion of an overpressured viscous shell of thickness $h$ is presented in Appendix A. By assuming the ideal gas law and isothermal gas conditions, initial internal bubble pressure, $P_{0}$, can be related to internal bubble pressure at some arbitrary time after depressurisation has occurred through the relation;

$$
P_{i} R^{3}=P_{0} R_{0}^{3}
$$

where $R_{0}$ is initial bubble radius. By fixing the volume of the shell of surrounding melt, $h$, and assuming the initial shell of melt has thickness significantly less than the radius of the bubble which it surrounds $\left(h_{0} \ll R_{0}\right)$, bubble radius can be expressed as;

$$
R^{2}=\frac{R_{0}^{2} h_{0}}{r}
$$

where $h_{0}$ is the initial thickness of the melt surrounding the pore and $r$ is the radial co-ordinate. By combining equations (11 13), a final expression which was used to model radial bubble growth was obtained ${ }^{47}$ :

$$
\frac{d R}{d t}=\frac{\left(\frac{P_{0} R_{0}^{3}}{R^{3}}-P_{e}\right) R^{4}}{12 \eta R_{0}^{2} h_{0}} .
$$




\section{Model Implementation \\ Solving the Heat Equation}

The initial particle temperature was assumed to be uniform throughout the particle and equal to the temperature of the particle's surrounding environment (the Unit 1 reactor). By imposing this initial condition and the aforementioned boundary conditions, equation (2) was solved using a dimensionless analytical solution for a convectively cooled sphere;

$$
\theta^{*}=\sum_{n=1}^{\infty} C_{n} \exp \left(-\zeta_{n}^{2} F o\right) \frac{1}{\zeta_{n} r^{*}} \sin \left(\zeta_{n} r^{*}\right)
$$

where $\theta^{*}$ is dimensionless temperature, $C_{n}$ and $\zeta_{n}$ are constants whose derivations are given in Appendix B. The dimensionless variables of temperature, $T$, radial position, $r$, and time, $t$, were defined, respectively, as;

$$
\begin{aligned}
& \theta^{*}=\frac{T-T_{\infty}}{T_{i}-T_{\infty}}, \\
& r^{*}=\frac{r}{r_{p}}, \\
& F o=\frac{k_{b} t}{\rho_{b} c_{b} r_{p}^{2}},
\end{aligned}
$$

where $T_{i}$ is the initial particle temperature and all other variables have been previously defined ${ }^{52}$.

\section{Solving the Bubble Growth Equation}

The radial void growth, given in equation (14), was calculated using the numerical Euler method. Iterations were performed over a time step, $d t$, of $10^{-4} \mathrm{~s}$ with a spatial resolution of $10^{-8} \mathrm{~m}$. Radial pore growth was terminated when the simulated viscosity of the melt surrounding the pore reached the glass transition temperature (the temperature at which the silicate melt changes to a polymer glass ${ }^{53}$ ), which was taken to be the temperature corresponding to a viscosity of $10^{12} \mathrm{~Pa} \cdot \mathrm{s}^{26}$.

\section{Simulating Particle Cooling}

Initially, the one dimensional radial and temporal temperature and viscosity profiles of a molten sphere of composition similar to a 'Type B' CsMP were calculated, as outlined above. Subsequently, 10 bubble nucleation sites were randomly selected along a one-dimensional grid which had a length equal to the simulated particle radius. A bubble was then modelled to grow radially outwards from each selected site using equation (14). The viscosity values corresponding to the initial nucleation site of each bubble were used as the viscosity of $h_{0}$. This simulation was then repeated for numerous radial 'probes' into the simulated particle until the produced modelled bubble volume was equal to that observed in 'Type B' CsMP. This simulation was performed over a range of initial temperatures and particle radii, and the void diameter distributions for each set of initial conditions was obtained.

\section{Data Availability}

The datasets generated and analysed during the current study are available in the GitHub repository; https://github.com/jackjamesturner/Modelling-the-Formation-of-Fukushima-Type-B-Ejecta .

\section{References}

1. Simons, M. et al. The 2011 magnitude 9.0 Tohoku-Oki earthquake: mosaicking the megathrust from seconds to centuries. Science 332, 1421-1435 (2011).

2. Martin, P. G. The 2011 Fukushima Daiichi Nuclear Power Plant accident. 2190-5061 (University of Bristol, Bristol, 2019).

3. Ranghieri, F. \& Ishiwatari, M., Learning from megadisasters : lessons from the great east Japan earthquake. (World Bank, 2014).

4. IAEA.The Fukushima Daiichi Accident. ISBN: 978-92-0-107015-9 (2015).

5. Tokyo Electric Power Company Incorporated. Fukushima nuclear accident analysis report. (2012).

6. Yanez, J., Kuznetsov, M. \& Souto-Iglesias, A., An analysis of the hydrogen explosion in the Fukushima-Daiichi accident. International Journal of Hydrogen Energy 40, 8261-8280 (2015). 
7. Luangdilok, W., The explosions at Fukushima Daiichi Unit 3 and Unit 4 and implications on the evaluation of 1F3 accident. Nuclear Engineering and Design 362, (2020).

8. Martin, P. et al., Project IPAD, a database to catalogue the analysis of Fukushima Daiichi accident fragmental release material, Scientific Data 7, (2020).

9. Steinhauser, G., Brandl, A. \& Johnson, T. E., Comparison of the Chernobyl and Fukushima nuclear accidents: a review of the environmental impacts, Science of the Total Environment 470-471, 800-817 (2014).

10. Kinoshita, N. et al., Assessment of individual radionuclide distributions from the Fukushima nuclear accident covering central-east Japan, Proceedings of the National Academy of Sciences 108, 19526-19529 (2011).

11. Igarashi, Y. et al., A review of Cs-bearing microparticles in the environment emitted by the Fukushima Dai-ichi Nuclear Power Plant accident, Journal of Environmental Radioactivity 205-206, 101-118 (2019).

12. Adachi, K., Kajino, M., Zaizen, Y., \& Igarashi, Y. Emission of spherical cesium-bearing particles from an early stage of the Fukushima nuclear accident, Scientific Reports 3, (2013).

13. Abe, Y. et al., Detection of uranium and chemical state analysis of individual radioactive microparticles emitted from the Fukushima nuclear accident using multiple synchrotron radiation X-ray analyses, Analytical Chemistry 86, 8521-8525 (2014).

14. Mukai, H. et al., Speciation of radioactive soil particles in the Fukushima contaminated area by IP autoradiography and microanalyses, Environmental Science and Technology 48, 13053-13059 (2014).

15. Furuki, G. et al., Caesium-rich micro-particles: A window into the meltdown events at the Fukushima Daiichi Nuclear Power Plant, Scientific Reports 7, (2017).

16. Satou, Y. et al., Analysis of two forms of radioactive particles emitted during the early stages of the Fukushima Dai-ichi Nuclear Power Station accident, Geochemical Journal 52, 137-143 (2018).

17. Chino, M. et al., Utilization of ${ }^{134} \mathrm{Cs} /{ }^{137} \mathrm{Cs}$ in the environment to identify the reactor units that caused atmospheric releases during the Fukushima Daiichi accident, Scientific Reports 6, (2016).

18. Nishihara, K., Iwamoto, H. \& Suyama, K. Estimation of fuel compositions in Fukushima-Daiichi nuclear power plant (in Japanese). Jaea 2012-018 (2012).

19. Miura, H. et al., Characterization of two types of cesium-bearing microparticles emitted from the Fukushima accident via multiple synchrotron radiation analyses, Scientific Reports 10, (2020).

20. Yoshida, N. \& Takahashi, Y., Land-surface contamination by radionuclides from the Fukushima Daiichi nuclear power plant accident, Elements 8, 201-206 (2012).

21. Martin, P. G. et al., Compositional and structural analysis of Fukushima-derived particulates using high-resolution $\mathrm{x}$-ray imaging and synchrotron characterisation techniques, Scientific Reports 10, (2020).

22. Martin, P. G., Satou, Y., Griffiths, I., Richards, D. \& and Scott, T., Analysis of External Surface Irregularities on Fukushima-Derived Fallout Particles, Frontiers in Energy Research 5, (2017).

23. Porritt, L. A., Russell, J. K. \& Quane, S. L., Pele's tears and spheres: Examples from Kilauea Iki, Earth and Planetary Science Letters 333-334, 171-180 (2012).

24. Klug, C., Cashman, K. \& Bacon, C., Structure and physical characteristics of pumice from the climactic eruption of Mount Mazama (Crater Lake), Oregon, Bulletin of Volcanology 64, 486-501 (2002).

25. Martin, P. G. et al., Provenance of uranium particulate contained within Fukushima Daiichi Nuclear Power Plant Unit 1 ejecta material, Nature Communications 10, (2019).

26. Benage, M. C. et al., Tying textures of breadcrust bombs to their transport regime and cooling history, Journal of Volcanology and Geothermal Research 274, 92-107 (2014).

27. Wright, H. M. N., Cashman, K. V., Rosi, M. \& R. Cioni, Breadcrust bombs as indicators of Vulcanian eruption dynamics at Guagua Pichincha volcano, Ecuador, Bulletin of Volcanology 69, 281-300 (2007).

28. Giachetti, T., Druitt, T. H., Burgisser, A., Arbaret, L. \& Galven, C., Bubble nucleation, growth and coalescence during the 1997 Vulcanian explosions of Soufrière Hills Volcano, Montserrat, Journal of Volcanology and Geothermal Research 193, 215-231 (2010).

29. Hall, M. L., Robin, C., Beate, B., Mothes, P. \& Monzier, M., Tungurahua Volcano, Ecuador: Structure, eruptive history and hazards, Journal of Volcanology and Geothermal Research 91, 1-21 (1999). 
30. Samaniego, P., le Pennec, J. L., Robin, C. \& Hidalgo, S., Petrological analysis of the pre-eruptive magmatic process prior to the 2006 explosive eruptions at Tungurahua volcano (Ecuador), Journal of Volcanology and Geothermal Research 199, 69-84 (2011).

31. Orsi, G. et al., A comprehensive study of pumice formation and dispersal: the Cretaio Tephra of Ischia (Italy), Journal of Volcanology and Geothermal Research 53, 329-354 (1992).

32. Giordano, D., Nichols, A. R. L. \& Dingwell, D. B., Glass transition temperatures of natural hydrous melts: A relationship with shear viscosity and implications for the welding process, Journal of Volcanology and Geothermal Research 142, 105-118 (2005).

33. Martin, P. G. et al., Structural and compositional characteristics of Fukushima release particulate material from Units 1 and 3 elucidates release mechanisms, accident chronology and future decommissioning strategy, Scientific Reports 10, 542-553 (2020).

34. Thomas, R. M. E. \& Sparks, R. S. J, Cooling of tephra during fallout from eruption columns, Bulletin of Volcanology 54, (1992).

35. Capaccioni, B. \& Cuccoli, F., Spatter and welded air fall deposits generated by fire-fountaining eruptions: Cooling of pyroclasts during transport and deposition, Journal of Volcanology and Geothermal Research 145, 263-280 (2005).

36. Morooka, K. et al., New highly radioactive particles derived from Fukushima Daiichi Reactor Unit 1: Properties and environmental impacts, Science of the Total Environment 773, (2021).

37. Giordano, D., Russell, J. K. \& Dingwell, D. B., Viscosity of magmatic liquids: A model, Earth and Planetary Science Letters 271, 123-134 (2008).

38. Cavcar, M. The International Standard Atmosphere (ISA), (Anadolu University, Turkey, 2000).

39. Past Weather in Fukushima, Japan — March 2011. Timeanddate.com https://www.timeanddate.com/weather/japan/fukushima/ historic?month=3\&year=2011 (2021). (Accessed: 30th April 2021)

40. Zheng, G. \& and List, R., Convective heat transfer of rotating spheres and spheroids with non-uniform surface temperatures, International Journal of Heat and Mass Transfer 39, 1815-1826 (1996).

41. Zwicker, D., py-pde: A Python package for solving partial differential equations, Journal of Open Source Software 5, (2020).

42. Jones, T. J., et al., Primary and secondary fragmentation of crystal-bearing intermediate magma, Journal of Volcanology and Geothermal Research 327, 70-83 (2016).

43. Rockwool ${ }^{\mathrm{TM}}$, How to Choose the Right Mineral Wool for Insulation. https://www.rockwool.com/siteassets/rw-sa/productdocumentation/corporate-collaterals/all/how-to-choose-the-right-mineral-wool-for-insulation.pdf?f=20201025055913. (Accessed: 22nd April 2021)

44. Aben, H. et al., On the extraordinary strength of Prince Rupert's drops, Applied Physics Letters 109, (2016).

45. World Health Organization, Ambient air pollution: a global assessment of exposure and burden of disease, Clean Air Journal 26, (2016) https://doi.org/10.17159/2410-972X/2016/v26n2a4.

46. SolidWorks. 3D CAD Design Software. Dassault Systèmes (2019).

47. Barclay, J., Riley, D. S. \& Sparks, R. S. J., Analytical models for pore growth during decompression of high viscosity magmas, Bulletin of Volcanology 57, 422-431 (1995).

48. Prousevitch, A. A., Sahagian, D. L. \& Anderson, A. T., Dynamics of diffusive pore growth in magmas: isothermal case, Journal of Geophysical Research 98, 22283-22307 (1993).

49. Okumura, T. et al., Reactor environment during the Fukushima nuclear accident inferred from radiocaesium-bearing microparticles, Scientific Reports 10, (2020).

50. Kogure, T. et al., Constituent elements and their distribution in the radioactive Cs-bearing silicate glass microparticles released from Fukushima nuclear plant, Microscopy 65, 451-459 (2016).

51. Hort, M. \& and Gardner, J., Constraints on cooling and degassing of pumice during Plinian volcanic eruptions based on model calculations, Journal of Geophysical Research: Solid Earth 105, 25981-26001 (2000).

52. Recktenwald, G., Transient, One-Dimensional Heat Conduction in a Convectively Cooled Sphere. (Portland State University, Oregon, 2006).

53. Meille, S. V. et al., Definitions of terms relating to crystalline polymers (IUPAC Recommendations 2011), Pure and Applied Chemistry 83, 1831-1871 (2011). 


\section{Acknowledgements}

This work was supported by funding from the Engineering and Physical Sciences Research Council (EPSRC) (Grant Reference: EP/S020659/1), with additional support received from the Japan Atomic Energy Agency (JAEA) Collaborative Laboratories for Advanced Decommissioning Sciences (CLADS).

\section{Author contributions statement}

L.C. and J.T. conceived and conducted the study, and produced the main manuscript. P.M. supervised the study. All authors reviewed the manuscript.

\section{Additional Information}

\section{Competing interests}

The authors declare no competing interests. 


\section{Supplementary Files}

This is a list of supplementary files associated with this preprint. Click to download.

- SupplementaryInformationAppendices.pdf 